\title{
The Karoubi envelope and Lee's degeneration of Khovanov homology
}

\author{
DROR BAR-NATAN \\ SCOTT MORRISON
}

\begin{abstract}
We give a simple proof of Lee's result from [5], that the dimension of the Lee variant of the Khovanov homology of a $c$-component link is $2^{c}$, regardless of the number of crossings. Our method of proof is entirely local and hence we can state a Lee-type theorem for tangles as well as for knots and links. Our main tool is the "Karoubi envelope of the cobordism category", a certain enlargement of the cobordism category which is mild enough so that no information is lost yet strong enough to allow for some simplifications that are otherwise unavailable.
\end{abstract}

57M25; 57M27, 18E05

\section{Introduction}

In a beautiful article [5], Eun Soo Lee introduced a second differential $\Phi$ on the Khovanov complex of a knot (or link) and showed that the resulting double complex has uninteresting homology. In a seemingly contradictory manner, this is a very interesting result - for this "degeneration" of the Lee theory is in itself an extra bit of information about the original Khovanov homology, masterfully used by Rasmussen [7] to define the aptly named "Rasmussen invariant" of a knot and to give a combinatorial proof of an old conjecture of Milnor.

Unfortunately Lee's proof of her degeneration result is a bit technical and inductive in nature. The purpose of this note is to reprove Lee's degeneration result in local terms, using tools in the spirit of [2]. Thus in addition to being a bit more conceptual, our methods work for tangles as well as for knots and links.

Another proof of Lee's result was found by S Wehrli [8]. His proof is quick, short and elegant; the only small advantage of our proof over his is its locality and applicability to tangles.

Let us sketch our results now; the relevant terminology (which closely follows [2]) will be quickly recalled in Section 2 below. 
A confluence within a smoothing $S$ of a tangle $T$ is a pair of arc segments in $S$ that correspond to a small neighbourhood of a crossing in $T$. Thus if $T$ has $n$ crossings, $S$ will have $n$ confluences; a crossing such as $\times$ in $T$ becomes a confluence such as ) ( or $\asymp$ in $S$.

Definition 1.1 Let $T$ be a tangle and let $S$ be a smoothing of $T$. An alternate colouring of $S$ is a colouring of the components of $S$ with two colours (always "red" and "green" below), so that the two arc segments at every confluence of $S$ are coloured with different colours.

Our main theorem is the following:

Theorem 1.2 Within the appropriate category (see below), the Khovanov-Lee complex of a tangle $T$ is homotopy equivalent to a complex with one generator for each alternately coloured smoothing of $T$ and with vanishing differentials.

Alternately coloured smoothings are easy to manage:

Proposition 1.3 Any $c$-component tangle $T$ has exactly $2^{c}$ alternately coloured smoothings. These smoothings are in a bijective correspondence with the $2^{c}$ possible orientations of the $c$ components of $T$.

Together Theorem 1.2 and Proposition 1.3 imply Lee's result that the Khovanov-Lee homology of an $c$-component link is $2^{c}$ dimensional.

What is that "appropriate category" of Theorem 1.2? It is the category of complexes over the "Karoubi envelope" $\operatorname{Kar}\left(\mathcal{C}_{o b_{1}}\right)$ of the category $\mathcal{C}_{o b_{1}}$ used to describe the Khovanov-Lee complex. It is perhaps the nicest gadget appearing in our note it introduces more objects into $\mathcal{C}_{o b} b_{1}$ allowing for more opportunities to simplify complexes over $\mathcal{C}_{o b}$, yet it does not introduce new morphisms between existing objects of $\mathcal{C} o b_{1}$, and hence no new homotopies or homotopy equivalences. Thus there is no loss of information in the passage from $\mathcal{C} o b_{1}$ to $\operatorname{Kar}\left(\mathcal{C}_{o b} b_{1}\right)$.

\subsection{Is there anything left to do?}

Plenty. Lee's degeneration is critical to understanding Rasmussen [7]. We have a clean approach to Lee's degeneration, and one may hope it will help improve our understanding of Rasmussen's work. This still remains to be done. More specifically, the Khovanov-Lee homology is filtered and the definition of the Rasmussen invariant requires this filtration. Thus:

Problem 1.4 Figure out how the filtration in the Khovanov-Lee homology interacts with everything down below. 


\subsection{The plan}

In Section 2 we quickly recall the relevant definitions of the Khovanov and KhovanovLee theories. Then in Section 3 we review the definition of the Karoubi envelope of a general category $\mathcal{C}$. Section 4 is the heart of the paper. In it we introduce the "red" and the "green" projections, the red-green splitting within $\operatorname{Kar}\left(\mathcal{C}_{o b_{1}}\right)$ of objects in $\mathcal{C}_{o b_{1}}$ and prove Theorem 1.2. Finally, in Section 5 we prove Proposition 1.3.

Acknowledgement We wish to thank S Wehrli for drawing our attention to [8] and A Referee for further comments and suggestions. This work was partially supported by NSERC grant RGPIN 262178.

\section{A quick review of the local Khovanov theory}

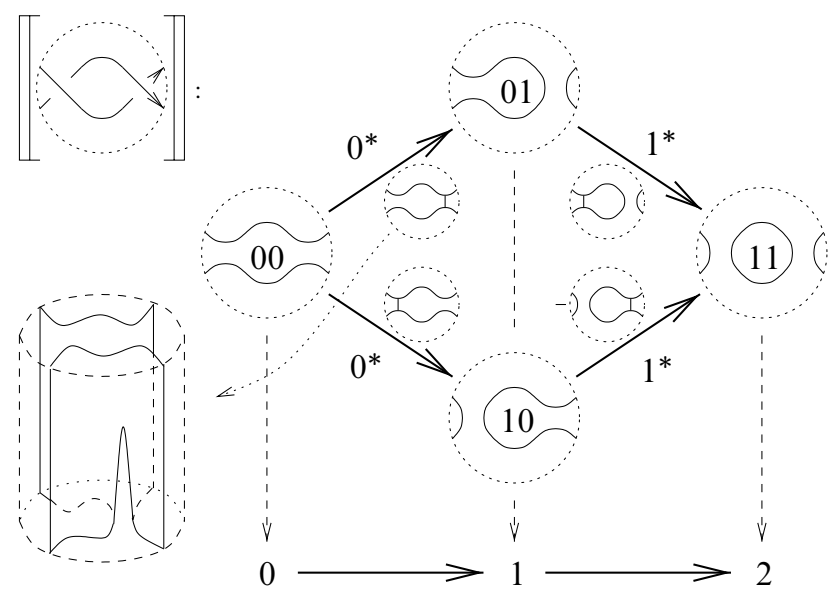

Figure 1: The Khovanov complex of a 2-crossing tangle

Let us briefly recall the definition of the Khovanov complex for tangles, following [2]. Given an $n$-crossing tangle $T$ with boundary $\partial T$ (such as the 2 -crossing tangle in Figure 1) one constructs an $n$-dimensional "cube" of 1 -dimensional smoothings and 2-dimensional cobordisms between them (as illustrated in Figure 1). This cube is then "flattened" to a "formal complex" $\llbracket T \rrbracket_{0}$ in the additive category $\mathcal{C}_{o} b_{0}(\partial T)$ (denoted $\mathcal{C} o b_{\bullet / l}^{3}(\partial T)$ in $\left.[1 ; 2]\right)$ whose objects are formally graded smoothings with boundary $\partial T$ and whose morphisms are formal linear combinations of cobordisms whose tops and bottoms are smoothings and whose side boundaries are $I \times \partial T$, modulo some 
local relations. An overall height shift ensures that the "oriented smoothing" appears in homological height 0 .

The Khovanov complex $\llbracket T \rrbracket_{0}$ is an object in the category $\operatorname{Kom}\left(\operatorname{Mat}\left(\operatorname{Cob}_{0}(\partial T)\right)\right)$ of complexes of formal direct sums of objects in $\mathcal{C}_{o b}(\partial T)$ and it is invariant up to homotopies.

For simplicity we are using as the basis to our story one of the simpler cobordism categories $\mathcal{C}_{o b}:=\mathcal{C}_{o b} b_{\bullet l}^{3}$ that appear in [2], rather than the most general one, $\mathcal{C}_{o b}^{3}$. It is worthwhile to repeat here the local relations that appear in the definition of $\mathcal{C o b}_{0}$ (see [2, Section 11.2]):
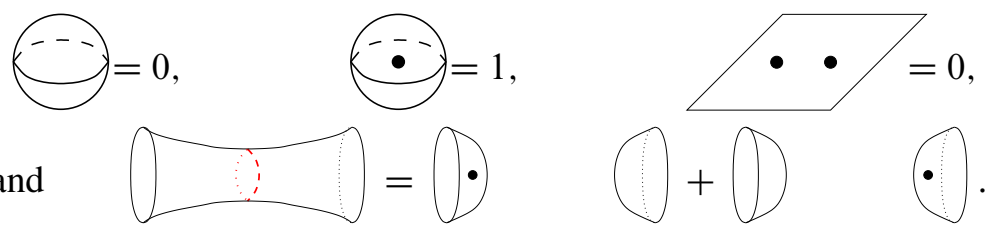

(If you're more used to the purely topological cobordism model without dots, recall the translation $\odot=\frac{1}{2} \omega$. Note that while one of the original motivations for describing the "dotted" theory was being able to work over $\mathbb{Z}$, we'll later need at least 2 to be invertible and so work over $\mathbb{Z}_{(2)}$.)

Also recall from [2, Section 5] that $\llbracket \cdot \rrbracket_{0}$ is a planar algebra morphism. That is, if $T_{1}$ and $T_{2}$ are tangles and $D\left(T_{1}, T_{2}\right)$ denotes one of their side-by-side compositions (a side by side placement of $T_{1}$ and $T_{2}$ while joining some of their ends in a certain way prescribed by a planar arc diagram $D)$, then $\llbracket D\left(T_{1}, T_{2}\right) \rrbracket_{0}=\llbracket T_{1} \rrbracket_{0} \otimes_{D} \llbracket T_{2} \rrbracket_{0}$. Here, as in [2, Section 5], $\llbracket T_{1} \rrbracket_{0} \otimes_{D} \llbracket T_{2} \rrbracket_{0}$ is the "tensor product" operation induced on formal complexes by the horizontal composition operation $D$.

The Lee variant of Khovanov homology, or the Khovanov-Lee complex $\llbracket T \rrbracket_{1}$ of a tangle $T$, is constructed in exactly the same way as $\llbracket T \rrbracket_{0}$; the only difference is that the zero in the third local relation in (2-1) is replaced with a one. Hence it is valued in the category of complexes over the category $\mathcal{C}_{o b} b_{1}(\partial T)$ defined in exactly the same manner as $\mathcal{C}_{0} b_{0}(\partial T)$, except with the following collection of local relations (spot the one difference!):



and
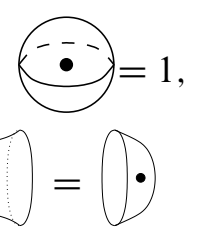

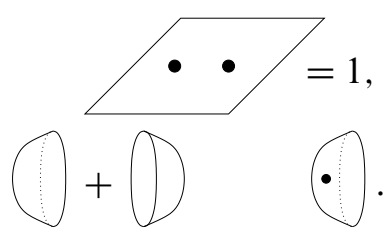


While $\mathcal{C}_{0} b_{0}$ can be made into a graded category (see [2, Section 6]), the degree of $\cdots \quad$ is nonzero, so setting it to 1 breaks the grading in $\mathcal{C}_{o} b_{1}$. Otherwise the Khovanov theory and the Khovanov-Lee theories are completely parallel. In particular, the Khovanov-Lee complex is also an up-to-homotopy knot invariant and it is also a planar algebra morphism.

\section{A quick review of the Karoubi envelope}

A projection is an endomorphism $p$ satisfying $p^{2}=p$. In many contexts in mathematics, if $p$ is a projection then so is $1-p$, and together these two projections decompose space as a direct sum of the image of $p$ with the image of $1-p$. Thus a projection often gives rise to a decomposition of space into pieces which are hopefully simpler.

The equation $p^{2}=p$ makes sense for an endomorphism in an arbitrary category, so projections make sense in an arbitrary category. And if $p$ is a projection then so is $1-p$ in an arbitrary additive category. But in a general (additive or not) category, "the image of $p "$ (or of $1-p$ ) may or may not make sense.

The Karoubi envelope $e^{1}$ of a category $\mathcal{C}$ is a way of adding objects and morphisms to $\mathcal{C}$ so that every projection has an image and so that if $p: \mathcal{O} \rightarrow \mathcal{O}$ is a projection and $\mathcal{C}$ is additive, then (with the proper interpretation) $\mathcal{O} \cong \operatorname{im} p \oplus \operatorname{im}(1-p)$. Thus sometimes complicated objects can be simplified in the Karoubi envelope of $\mathcal{C}$, while in $\mathcal{C}$ they may be indecomposable.

Let us turn to the formal definitions.

Definition 3.1 Let $\mathcal{C}$ be a category. An endomorphism $p$ : $\mathcal{O} \rightarrow \mathcal{O}$ of some object $\mathcal{O}$ in $\mathcal{C}$ is called a projection if $p \circ p=p$. The Karoubi envelope $\operatorname{Kar}(\mathcal{C})$ of $\mathcal{C}$ is the category whose objects are ordered pairs $(\mathcal{O}, p)$ where $\mathcal{O}$ is an object in $\mathcal{C}$ and $p: \mathcal{O} \rightarrow \mathcal{O}$ is a projection. If $\left(\mathcal{O}_{1}, p_{1}\right)$ and $\left(\mathcal{O}_{2}, p_{2}\right)$ are two such pairs, the set of morphisms in $\operatorname{Kar}(\mathcal{C})$ from $\left(\mathcal{O}_{1}, p_{1}\right)$ to $\left(\mathcal{O}_{2}, p_{2}\right)$ is the collection of all $f: \mathcal{O}_{1} \rightarrow \mathcal{O}_{2}$ in $\mathcal{C}$ for which $f=f \circ p_{1}=p_{2} \circ f$. An object $(\mathcal{O}, p)$ in $\operatorname{Kar}(\mathcal{C})$ may also be denoted by $\operatorname{im} p .^{2}$

The composition of morphisms in $\operatorname{Kar}(\mathcal{C})$ is defined in the obvious way (by composing the corresponding $f$ 's). The identity automorphism of an object $(\mathcal{O}, p)$ in

\footnotetext{
${ }^{1}$ The Karoubi envelope construction [10] was first described by Freyd [3], a few years before Karoubi. It has previously been used in motivic cohomology by Mazur [6] and in diagrammatic representation theory, eg Kuperberg [4].

${ }^{2}$ If you're worried about just introducing im $p$ as notation, when you already know a category-theoretic definition of image, eg [9], don't be; this is actually an image.
} 
$\operatorname{Kar}(\mathcal{C})$ is $p$ itself. It is routine to verify that $\operatorname{Kar}(\mathcal{C})$ is indeed a category. There is an obvious embedding functor $\mathcal{I}: \mathcal{O} \mapsto(\mathcal{O}, I)$ of $\mathcal{C}$ into $\operatorname{Kar}(\mathcal{C})$ and quite clearly, $\operatorname{mor}_{\operatorname{Kar}(\mathcal{C})}\left(\mathcal{I O}_{1}, \mathcal{I} \mathcal{O}_{2}\right)=\operatorname{mor}_{\mathcal{C}}\left(\mathcal{O}_{1}, \mathcal{O}_{2}\right)$ for any pair of objects $\mathcal{O}_{1,2}$ in $\mathcal{C}$. Thus we will simply identify objects in $\mathcal{C}$ with their image via $\mathcal{I}$ in $\operatorname{Kar}(\mathcal{C})$.

Below we will assume that $\mathcal{C}$ is an additive category and that direct sums of objects make sense in $\mathcal{C}$. As in [2], there is no loss of generality in making these assumptions as formal sums of morphisms and formal direct sums of objects may always be introduced.

Proposition 3.2 Let $p: \mathcal{O} \rightarrow \mathcal{O}$ be an endomorphism in $\mathcal{C}$.

(1) If $p$ is a projection then so is $1-p$.

(2) In this case, $\mathcal{O} \cong \operatorname{im} p \oplus \operatorname{im}(1-p)$ in $\operatorname{Kar}(\mathcal{C})$.

\section{Proof}

(1) $(1-p)^{2}=1-2 p+p^{2}=1-2 p+p=1-p$ (sorry for the damage to the rainforest).

(2) The isomorphism $\mathcal{O} \rightarrow \operatorname{im} p \oplus \operatorname{im}(1-p)$ is given by the $1 \times 2$ matrix $(p 1-p)$. Its inverse is the $2 \times 1$ matrix $\left(\begin{array}{c}p \\ 1-p\end{array}\right)$.

Observe that if $p$ is a projection on $\mathcal{O}$ and $p^{\prime}$ is a projection on $\mathcal{O}^{\prime}$, then the set $\operatorname{Hom}\left((\mathcal{O}, p),\left(\mathcal{O}^{\prime}, p^{\prime}\right)\right)$ may be naturally identified with $p^{\prime} \operatorname{Hom}\left(\mathcal{O}, \mathcal{O}^{\prime}\right) p$. In fact, even before taking the Karoubi envelope, $\operatorname{Hom}\left(\mathcal{O}, \mathcal{O}^{\prime}\right)$ can be expressed as a direct sum of 4 "matrix entries", each obtained by precomposing with $p$ or $1-p$, and postcomposing with $p^{\prime}$ or $1-p^{\prime}$.

In this paper we are mainly interested in complexes whose "chain spaces" are objects in some category $\mathcal{C}$ as above. The previous proposition tells us that there may be some gain by switching to working over $\operatorname{Kar}(\mathcal{C})$ as we may have new decompositions of old objects. The proposition below tells us that there is no loss of information in doing so.

Proposition 3.3 Let $\Omega_{1}$ and $\Omega_{2}$ be complexes in $\operatorname{Kom}(\mathcal{C})$. If $\Omega_{1}$ and $\Omega_{2}$ are homotopy equivalent as complexes in $\operatorname{Kom}(\operatorname{Kar}(\mathcal{C}))$, they were already homotopy equivalent as complexes in $\operatorname{Kom}(\mathcal{C})$.

Proof A homotopy equivalence $\Upsilon$ in $\operatorname{Kom}(\operatorname{Kar}(\mathcal{C}))$ between $\Omega_{1}$ and $\Omega_{2}$ is a certain slew of morphisms between objects appearing in $\Omega_{1}$ and objects appearing in $\Omega_{2}$ (a chain morphism going one way, another going the other way and a couple of homotopies). All those morphisms are between objects in $\mathcal{C}$ and as noted above, $\operatorname{Kar}(\mathcal{C})$ introduces no new morphisms between objects in $\mathcal{C}$. So $\Upsilon$ is really in $\mathcal{C}$. 


\section{Red and green in Lee's theory}

Viewed from our perspective, the key to Lee's theorem is the presence in $\mathcal{C}_{o b} b_{1}$ of two complementary projections, the red projection $r$ and the green projection $g$, that can be composed both vertically (in the "category" direction of $\mathcal{C} o b_{1}$ ) and horizontally (in the "planar algebra" direction; see [2, Section 8.2]). Let us start with some elementary school algebra that contains all the calculations we will need regarding $r$ and $g$.

Lemma 4.1 Let $b$ (for $\backslash$ bullet) be a variable satisfying $b^{2}=1$ (find it in (2-2)!), let $r$ (for red) be $(1+b) / 2$ and let $g$ (for green) be $(1-b) / 2$. Then $r$ and $g$ are the eigenprojections associated to the involution $b$. In particular,

(1) $r$ and $g$ are projections: $r^{2}=r$ and $g^{2}=g$.

(2) $r$ and $g$ are complementary: $r+g=1$.

(3) $r$ and $g$ are disjoint: $r g=0$.

(4) $r$ and $g$ are eigenprojections of $b: b r=r$ and $b g=-g$.

In $\mathcal{C} o b_{1}$, one may place a $b$ (ie a bullet) anywhere on any cobordism and thus, treating linear combinations in the obvious manner, one may place an $r$ or a $g$ anywhere on any cobordism. In particular, we may place them on any "vertical curtain", ie on any connected component of the identity morphism from a smoothing to itself. The resulting "identities labeled $r$ and/or $g$ " are projections by the above lemma and hence they represent objects in the Karoubi envelope $\operatorname{Kar}\left(\mathcal{C}_{o b_{1}}\right)$ of $\mathcal{C} o b_{1}$. Thus in $\operatorname{Kar}\left(\mathcal{C}_{o b_{1}}\right)$ we have, for example, the isomorphism

$$
\left.\left.\sim \sim\right|_{r} \oplus\right|_{g}
$$

between a single arc smoothing and a direct sum of two single arc smoothings, one paired with the $r$ projection and one with $g$. Likewise we also have the isomorphism $(4-1)$

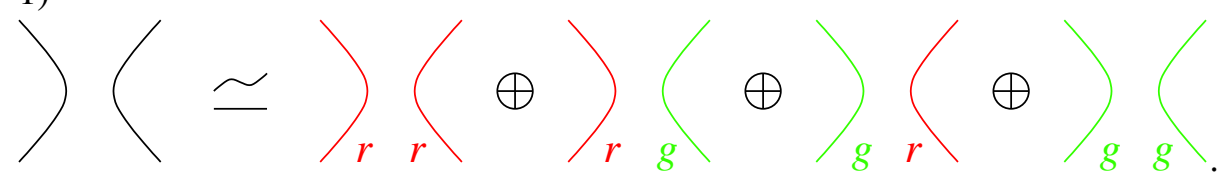

We can now observe that a dramatic simplification occurs at the very first step of calculating a knot invariant; the complex associated to a single crossing now has an up-to-homotopy representative with a vanishing differential. Indeed, $[[\times]]_{1}$ is the 
two-step complex $)(\stackrel{H}{\longrightarrow} \asymp$ in which $)$ ( denotes the saddle morphism. As in (4-1), each of the two objects ()$($ and $\asymp)$ appearing in this complex becomes a direct sum of four objects in $\operatorname{Kar}\left(\mathcal{C}_{o b_{1}}\right)$. The differential $)($ becomes a $4 \times 4$ matrix $M$ all of whose entries are saddles, and each such saddle carries $r$ and $g$ insertions to match the colourings of its domain and target smoothings. But $r$ and $g$ are disjoint (see Lemma 4.1) and the saddle cobordism is connected, so a saddle bearing insertions of more than one colour vanishes and hence only two of the 16 entries of $M$ survive. Thus in $\operatorname{Kom}\left(\operatorname{Kar}\left(\operatorname{Cob}_{1}\right)\right)$,

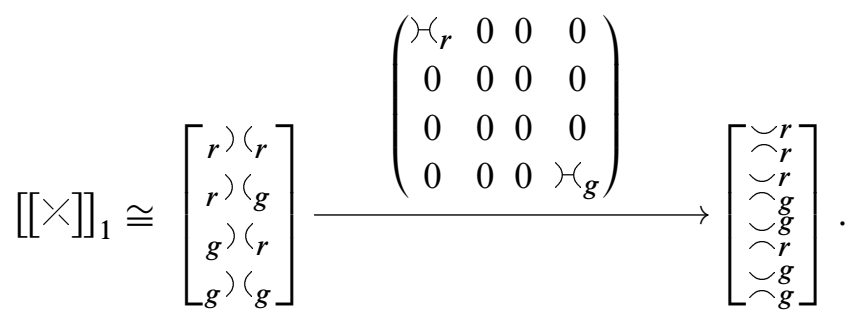

This last complex is a direct sum of four complexes. The first and the last of the four summands,

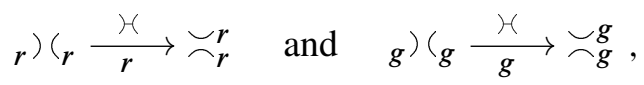

are contractible as $)_{r}$ and $)_{g}$ are invertible (with inverses $\frac{1}{2} \nearrow_{r}$ and $-\frac{1}{2} \nearrow_{g}$, respectively) ${ }^{3}$. Thus up to homotopy,

$$
[[\times]]_{1} \simeq\left[\begin{array}{l}
r)(g \\
g)(r
\end{array}\right] \stackrel{0}{\longrightarrow}\left[\begin{array}{l}
\smile r \\
\hdashline g \\
\smile g \\
\smile r
\end{array}\right]
$$

and as promised, we found a representative for $[[\times]]_{1}$ with a vanishing differential.

Proof of Theorem 1.2 Theorem 1.2 is now simply a matter of assembling the pieces. The discussion above shows that it holds for tangles consisting of a single crossing.

If we build a tangle $T$ by combining crossings $X_{1}$ through $X_{n}$ using a planar operation $D$, then $\llbracket T \rrbracket_{1}$ is the tensor product $\bigotimes_{D} \llbracket X_{i} \rrbracket_{1}$.

Since the complexes $\llbracket X_{i} \rrbracket_{1}$ have an object for each of the four alternately coloured smoothing of $X_{i}$ and no nonzero differentials, the complex $\llbracket T \rrbracket_{1}$ also has no differentials, but at first sight too many objects. While every alternately coloured smoothing of $T$ appears as an object (because an alternately colouring smoothing can be divided into alternating coloured smoothings of the constituent crossings), we also have smoothings

\footnotetext{
${ }^{3}$ This follows from the neck cutting relation (the last of (2-2)) and the final part of Lemma 4.1.
} 
which are alternately coloured, but have different colours appearing on arcs which are connected. Now remember that Lemma 4.1 holds for horizontal compositions as well as vertical ones. A strand with both colours inserted is the same as a strand with the zero projection inserted and so is equivalent in the category to the zero object; the extra objects all disappear.

\section{Alternately coloured smoothings and orienting components}

In order to return a little closer to Lee's language in [5], we will now show that the alternately coloured smoothings of Section 4 are in a one-to-one correspondence with orientations of the original tangle.

Proof of Proposition 1.3 To begin, consider a tangle diagram $T$ and give its regions a red-green checkerboard colouring. (The region outside the tangle disk doesn't receive a colour; further, let's agree that the outer region of a knot, or the marked boundary region of a tangle, is green.)

We can canonically associate to an orientation of $T$ the oriented smoothing, in which each arc is consistently oriented. We now need to produce colours for the resulting arcs. As the arcs remain oriented, we can simply take the colour appearing in the region on the right. That this is consistent follows from the observation that as you pass through a crossing, switching from one strand to the other, the checkerboard colours appearing to your left and right remain the same. Further, the colouring we've produced alternates near each confluence, because as two incoming strands enter a crossing they have opposite colours to their right.

Conversely, from an alternately coloured smoothing of a tangle, we need to define an orientation of the tangle. Each arc of the knot has been coloured either red or green, and it has a red and a green region on either side. We orient the arc so that its own colour appears on its right. Moving from one arc of the tangle to another through a crossing, the colour of the arc changes, because the smoothing is alternately coloured, and at the same time the checkerboard colourings on either side switch. This ensures that two opposite arcs at a crossing receive consistent orientations.

These two constructions are clearly inverses, so we have the desired bijection. The four orientations of the Whitehead link and the corresponding alternately coloured smoothings are shown in Figure 2.

Without any difficulty, we can also describe the homological height of each generator. For this we need to compare a chosen orientation of our tangle with the fixed "original" 

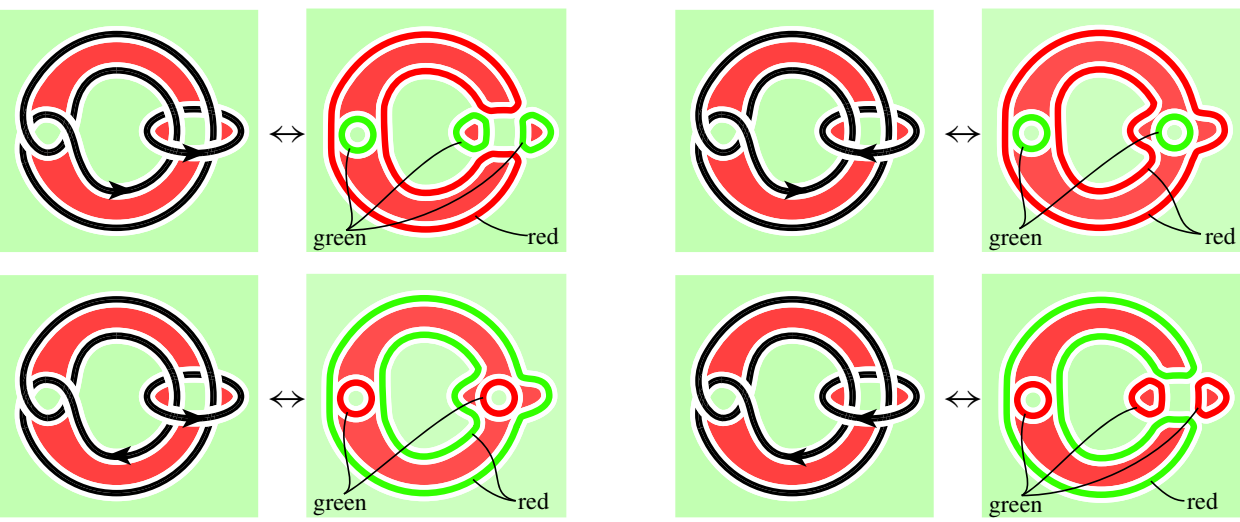

Figure 2: Generators of the Khovanov-Lee homology of the Whitehead link. (In greyscale, replace "red" and "green" with "dark" and "light".) All four generators are in homological height zero, because the two components have linking number 0 (see Proposition 5.1).

orientation (recall that the orientation of a tangle is required in the definition of the Khovanov homology to fix an overall homological height shift). Split the tangle into two parts; $T_{+}$containing those components where the chosen and fixed orientations agree and $T_{-}$containing the components where the orientations disagree.

Proposition 5.1 Considering $T_{+}$and $T_{-}$to carry the original orientations, the homological height of the corresponding generator is $\operatorname{lk}\left(T_{+}, T_{-}\right)$.

Proof This holds for the two component tangle consisting of a single crossing. If both strands carry the original orientation, or both carry the opposite orientation, then one of $T_{+}$and $T_{-}$is empty, so the linking number is zero. The oriented smoothing of this crossing is also the oriented smoothing with respect to the original orientations and so sits in homological height zero. If exactly one of the strands has been reversed, $1 \mathrm{k}\left(T_{+}, T_{-}\right)= \pm 1$, agreeing with the sign of the crossing (in the original orientation). Happily, the oriented smoothing of the crossing is actually the unoriented smoothing with respect to the original orientations, and so sits in homological height \pm 1 , again depending on the sign of the crossing.

To extend the result to arbitrary tangles, it suffices to note that every generator of $\llbracket T \rrbracket_{1}$ is a planar composition of generators for crossings and that both $1 \mathrm{k}\left(T_{+}, T_{-}\right)$and homological height are additive under planar composition. 


\section{References}

[1] D Bar-Natan, Fast Khovanov Homology Computations, http: //www . math.toronto.edu/ drorbn/papers/FastKh/

[2] D Bar-Natan, Khovanov's homology for tangles and cobordisms, Geom. Topol. 9 (2005) 1443-1499 MR2174270

[3] P Freyd, Abelian categories. An introduction to the theory of functors, Harper's Series in Modern Mathematics, Harper \& Row Publishers, New York (1964) MR0166240

[4] G Kuperberg, Spiders for rank 2 Lie algebras, Comm. Math. Phys. 180 (1996) 109151 MR1403861

[5] ES Lee, An endomorphism of the Khovanov invariant, Adv. Math. 197 (2005) 554-586 MR2173845

[6] B Mazur, What is . . a motive?, Notices Amer. Math. Soc. 51 (2004) 1214-1216 MR2104916

[7] J A Rasmussen, Khovanov homology and the slice genus arXiv:math.GT/0402131

[8] SM Wehrli, A spanning tree model for Khovanov homology arXiv: math.GT/0409328

[9] Wikipedia, Image (category theory) - Wikipedia, The Free Encyclopedia, http://en.wikipedia.org/w/index.php?title=Image_(category_theory) (2006)

[Online; accessed 20-June-2006]

[10] Wikipedia, Karoubi envelope - Wikipedia, The Free Encyclopedia, http://en.wikipedia.org/w/index.php?title=Karoubi_envelope (2006) [Online; accessed 20-June-2006]

Department of Mathematics, University of Toronto

Toronto Ontario M5S 2E4, Canada

Department of Mathematics, University of California, Berkeley

Berkeley CA 94720, USA

drorbn@math.toronto.edu, scott@math.berkeley.edu

http://www.math.toronto.edu/ drorbn, http://math. berkeley.edu/ scott

Received: 29 June 2006

Algebraic $6 \mathcal{G}$ Geometric Topology, Volume 6 (2006) 